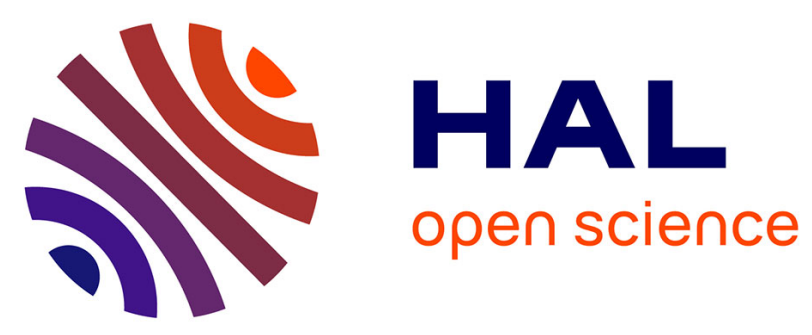

\title{
OTOÉMISSIONS ET AUDIOMÉTRIE DES TRAUMATISMES ACOUSTIQUES : CONSÉQUENCES POUR LES MODĖLES DE GENĖSE D'OTOÉMISSIONS
}

\author{
P. Avan, D. Loth, M. Teyssou, C. Menguy
}

\section{To cite this version:}

P. Avan, D. Loth, M. Teyssou, C. Menguy. OTOÉMissions ET AUDIOMÉTRIE DES TRAUMATISMES ACOUSTIQUES: CONSÉQUENCES POUR LES MODĖLES DE GENĖSE D'OTOÉMISSIONS. Journal de Physique IV Proceedings, 1992, 02 (C1), pp.C1-169-C1-172. 10.1051/jp4:1992133 . jpa-00251204

HAL Id: jpa-00251204 https://hal.science/jpa-00251204

Submitted on 1 Jan 1992

HAL is a multi-disciplinary open access archive for the deposit and dissemination of scientific research documents, whether they are published or not. The documents may come from teaching and research institutions in France or abroad, or from public or private research centers.
L'archive ouverte pluridisciplinaire HAL, est destinée au dépôt et à la diffusion de documents scientifiques de niveau recherche, publiés ou non, émanant des établissements d'enseignement et de recherche français ou étrangers, des laboratoires publics ou privés. 


\title{
OTOEMISSIONS ET AUDIOMETRIE DES TRAUMATISMES ACOUSTIQUES : CONSEQUENCES POUR LES MODÈLES DE GENÈSE D'OTOÉMISSIONS
}

\author{
P. AVAN, D. LOTH, M. TEYSSOU et C. MENGUY \\ Département de Biophysique (P.A.M. Neuro-sensoriel), Faculté de Médecine Lariboisière, Universiué \\ Paris VII, 10 avenue de Verdun, F-75010 Paris, France
}

\begin{abstract}
:
The amplitudes and detection thresholds of click-evoked otoacoustic emissions (c-EOE) and SFE were determined in 58 patients suffering from acoustic trauma. A multivariate statistical analysis was performed in order to investigate the relationships between EOE around $0.75,1$ and $2 \mathrm{kHz}$, and the various audiometric thresholds. Only one significant correlation was found between $E O E$ threshold at $F$ and auditory threshold at $2 F$, suggesting that some EOE properties are not frequency-specific. It is shown that the alternative hypothesis assuming that $E O E$ amplitudes are proportional to the total number of residual active sites along the basal cochlea provides a much better fit to experimental data and is consistent with several models and experimental results.
\end{abstract}

\section{Introduction}

La sensibilité et la sélectivité fréquentielle des récepteurs cochléaires repose sur le fonctionnement des cellules ciliées externes (CCE) de l'organe de Corti, et notamment sur l'existence dans ces cellules d'une transduction bidirectionnelle [1]. L'activité électro-mécanique normale des CCE est à l'origine des différents types d'otoémissions acoustiques (OEA), dont la détection est devenue un outil de dépistage senisible des lésions de ces cellules. En ce qui concerne les OEA provoquées (par clic, bouffée tonale ou son pur continu), la présence ou l'absence de réponses permet de séparer les sujets en 2 catégories de part et d'autre d'un seuil de perte auditive de l'ordre de $30 \mathrm{~dB}[2,3]$. Il s'agit donc d'une analyse qualitative et globale voire même binaire. Les autres approches se heurtent à la difficulté d'analyser des résultats apparemment entachés d'une grande variance inter-individuelle. Si l'importance de ces approches est moindre sur le plan clinique, où la seule application à part entière des OEÁ est le dépistage des nourrissons [3], il est en revanche important sur le plan fondamental d'approfondir les mécanismes de genèse et de propagation de ces phénomènes, afin de répondre aux questions suivantes: (1) Existe-t-il une corrélation significative entre audiogramme tonal et spectre fréquentiel des OEA? (2) Si cette corrélation existe, signifie-t-elle que les OEA sont le reflet des propriétés purement locales de la cochlée? (3) Sinon, comme les phénomènes de genèse et de propagation des OEA ne peuvent être aisément dissociés dans les différents modèles de mécanique cochléaire, la propagation des OEA peut-elle être affectée par l'état global de la cochlée?

\section{Objectif de ce travail, matériel et méthodes}

La première étape de ce travail a été de déterminer les amplitudes, caractéristiques d'entrée/sortie et seuils de détection des OEA provoquées par clic et des OEA synchrones d'un son continu (également dénommées SFE c'est-à-dire 'stimulus frequency emissions') dans 58 cas de traumatismes acoustiques. Dans 30 cas, il s'agissait de traumatismes acoustiques récents dus à des bruits d'arme à feu, chez des sujets jeunes sans antécédent otologique. Les autres cas avaient subi des expositions répétées donc étaient porteurs de lésions plus anciennes. Pour chacun des sujets (volontaires), une audiométrie tonale et une audiométrie automatique de Békésy ont été pratiquées, l'absence d'atteinte transmissionnelle ou rétro-cochléaire a été vérifiée. 
L'enregistrement des OEA provoquées par clic a utilisé un appareil ILO88 (Otodynamics) [4] et celui des SFE une chaîne de détection précédemment décrite [2]. L'analyse des OEA a été pratiquée à plusieurs fréquences bien déterminées, $750 \mathrm{~Hz}, 1$ et $2 \mathrm{kHz}( \pm 100 \mathrm{~Hz})$. Le stimulus était présenté à plusieurs niveaux, le premier élevé pour vérifier la non-linearité des réponses (soit $40 \mathrm{~dB}$ HL pour un clic), les autres niveaux étant décroissants par pas de $5 \mathrm{~dB}$. Le seuil de détection d'une OEA à une fréquence donnée est le plus petit niveau de stimulation permettant la détection fiable de lOEA au dessus du bruit de fond (maintenu constant d'un sujet à l'autre soit $-25 \mathrm{~dB}$ SPL/bande de $50 \mathrm{~Hz}$ ). Cette détermination est précise à $5 \mathrm{~dB}$ près et permet de caractériser la courbe d'entrée/sortie des OEA dans sa partie linéaire. Les données obtenues pour les SFE permettent de définir un seuil équivalent d'OEA et sont ensuite traitées de manière analogue [2].

Une première analyse des données d'audiométrie tonale a montré que les seuils audiométriques sont des variables étroitement corrélées. De ce fait, une corrélation trouvée entre les seuils de détection des OEA et les seuils auditifs à la même fréquence serait dépourvue de signification. Une analyse multivariée ('Stepwise regression) est donc indispensable pour rechercher les relations entre seuils de détection d'OEA et seuils auditifs en sons purs (par pas d'une demi-octave entre 250 et $8000 \mathrm{~Hz}$ ).

\section{Résultats}

Le modèle statistique obtenu est particulièrement simple mais déconcertant: une seule corrélation, d'ailleurs très significative ( $\mathrm{p}<0.001)$, apparaît et relie linéairement le seuil d'OEA à $\mathrm{N}$ kHz et le seuil auditif $2 \mathrm{~N} \mathrm{kHz}$ (fig.1). Ce modèle est indépendant de la technique utilisée; même les SFE, utilisant un stimulus spécifique en frequence, ont ce comportement. Il existe donc un décalage d'une octave environ entre les modifications pathologiques de l'audiogramme et celles des OEA au voisinage de leur seuil de détection. Il est remarquable que lorsqu'une analyse similaire est réalisée pour l'unique donnée habituellement retenue en clinique, c'est-à-dire présence ou absence d'OEA dans un intervalle fréquentiel donné, la meilleure corrélation trouvée devient spécifique en fréquence, donc relie une OEA de fréquence donnée et le seuil audiométrique à cette même fréquence (par exemple, fig. 1 à gauche). Cette dernière constatation est déjà l'objet de nombreuses publications [revue dans 4].
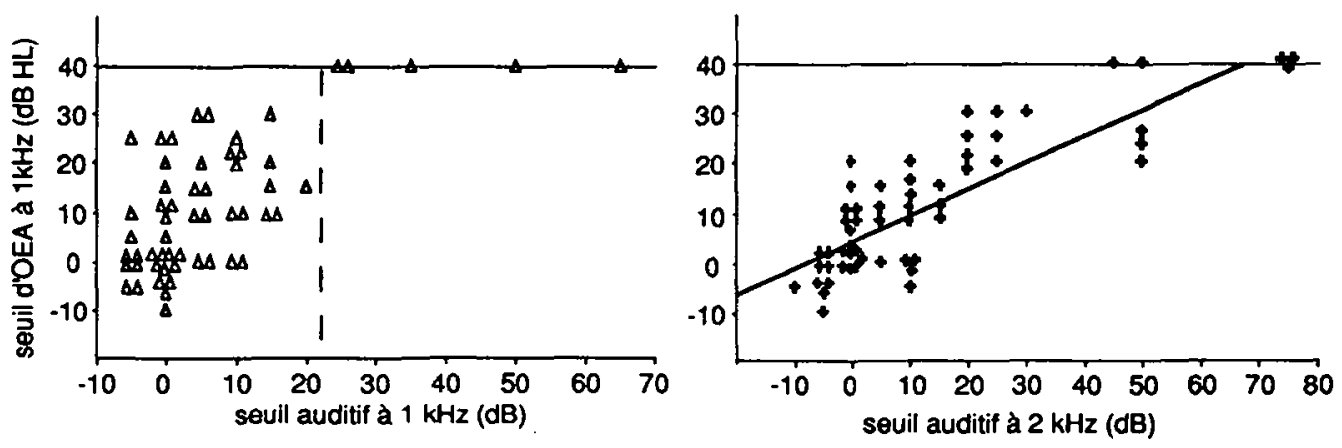

Figure 1: Le seuil de détection des OEA de fréquence $1 \mathrm{kHz}$ est représenté en fonction du seuil auditif à 1 $\mathrm{kHz}$ (à gauche) et $2 \mathrm{kHz}$ (à droite). Seule le graphique de droite présente une corrélation significative. Lorsqu'aucune OEA n'est détectable au voisinage de $1 \mathrm{kHz}$, le seuil de détection est arbitrairement fixé à $40 \mathrm{~dB}$ (5 cas).

Il est délicat de tirer des conclusions physiologiques d'un modèle statistique multivarie, même sur une population nombreuse comme dans ce travail. La seule conclusion indiscutable est négative: l'absence de toute corrélation réelle entre amplitude d'une OEA (provoquée par clic ou son continu) et seuil audiométrique à la même fréquence.

L'étape suivante de l'analyse des données repose donc sur l'hypothèse alternative, selon laquelle les propriétés d'une OEA détectée dans le conduit auditif externe dépend de l'état global de la cochlée (bien sûr seule la cochlée basale est considérée, puisque la propagation intra-cochléaire d'une vibration acoustique de frequence $F$ n'intéresse pratiquement pas la cochlée apicale par rapport au point de 
fréquence caractéristique F). Cette analyse consiste d'abord à mesurer la longueur de cochlée basale non lésée soit L(F) (estimée sur l'audiogramme de Békésy, comme la largeur -en fraction d'octaves- de l'intervalle entre $F$ et $8 \mathrm{kHz}$ dans lequel le seuil auditif est $<15 \mathrm{~dB} \mathrm{HL}$ ). Le seuil de détection de l'OEA de fréquence $F$, soit $S(F)$, est représenté en fonction de $L(F)$ et une analyse statistique est réalisée (fig.2). La régression linéaire obtenue est très proche de la relation mathématique suivante:

$$
\Delta S(F)=20 \times \log \left[L(F) / L_{0}(F)\right]
$$

$\triangle S$ en $\mathrm{dB}, \mathrm{L}(\mathrm{F})$ en octaves

$\mathrm{L}(\mathrm{F})=\mathrm{L}_{0}(\mathrm{~F})$ pour une oreille normale,

soit par exemple 3 octaves pour $\mathrm{F}=1 \mathrm{kHz}$ ou 2 octaves pour $\mathrm{F}=2 \mathrm{kHz}$

Autrement dit, le seuil de détection d'une OEA (par clic ou son continu), dépend linéairement de la largeur de cochlée intacte basale par rapport à l'emplacement accordé à la fréquence étudiée, quelque soit la forme de l'audiogramme tonal. Par conséquent, le modèle theorique, basé sur l'hypothèse selon laquelle les seuils de détection et amplitudes d'OEA ne sont pas spécifiques en fréquence mais au contraire proportionnels au nombre total de sites "actifs" le long de la cochlé basale, permet le meilleur ajustement des données statistiques.

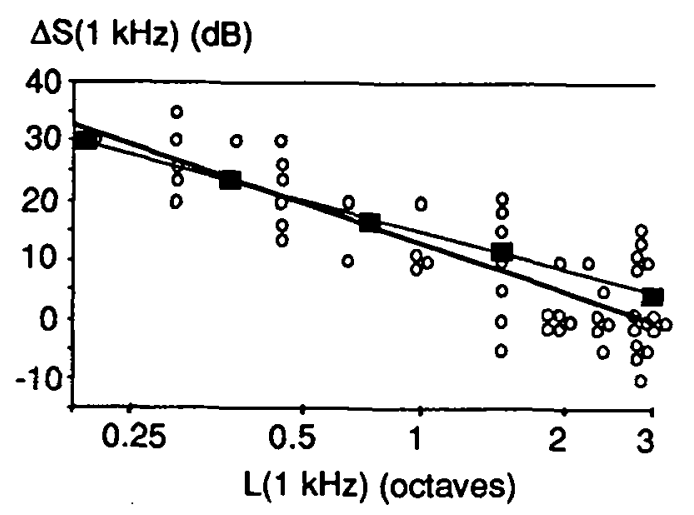

Figure 2: Les mêmes seuils de détection que sur la figure 1 sont maintenant représentés en fonction de la longueur de cochlée résiduelle. La droite de régression est en trait épais $\left(R^{2}=0,68\right)$; la droite en trait fin et les carrés noirs représentent les prédictions du modele thérique simplifié (équation 1).

\section{Discussion}

L'existence de ces relations permet de mieux comprendre la présence d'une corrélation privilégiée entre seuil de détection d'une OEA de fréquence $F$ et seuil auditif a $2 F$. En effet, il suffit d'appliquer la relation (1) au cas le plus fréquent des audiogrammes de forme régulière, c'est-à-dire pour lesquels la perte auditive dépasse toujours $15 \mathrm{~dB}$ au dessus d'une fréquence donnée, qui diminue lorsque la surdité s'aggrave. Prenons par exemple $F=1 \mathrm{kHz}$. Compte tenu de la sensibilité des mesures, l'élévation du seuil de détection de l'OEA à $1 \mathrm{kHz}$ ne devient détectable que lorsqu'elle dépasse $10 \mathrm{~dB}$ par rapport à la moyenne de la population normale soit $5 \mathrm{~dB} \mathrm{HL}$ avec le même appareillage [2]. Il faut donc $\mathrm{L}(\mathrm{F}) \approx 1$ octave ce qui correspond à un début d'atteinte à $2 \mathrm{kHz}$. Lorsque l'atteinte progresse, le seuil audiométrique varie a $2 \mathrm{kHz}$, mais reste longtemps normal a $1 \mathrm{kHz}$, et le seuil de détection de lOEA à 1 $\mathrm{kHz}$ varie parallèlement au seuil auditif a $2 \mathrm{kHz}$. Il faut toutefois noter que l'OEA à $1 \mathrm{kHz}$ finit par ne plus être détectable, pratiquement lorsque les lésions atteignent la fréquence audiométrique $1 \mathrm{kHz}$. Tous seuils de détection confondus, la présence de l'OEA à $1 \mathrm{kHz}$ est alors bien corrélée au seuil auditif à la même fréquence. Ceci peut rendre compte des relations parfois constatées entre les allures du spectre frequentiel des OEA et de l'audiogramme tonal.

L'hypothèse selon laquelle les seuils de détection et amplitudes d'OEA ne sont pas spécifiques en fréquence mais au contraire proportionnels (très schématiquement) au nombre total de sites "actifs" le long de la cochlée basale, est sous-jacente à un grand nombre de modèles théoriques décrivant la 
propagation des vibrations acoustiques le long de la membrane basilaire $[1,5,6,7,8]$. En conclusion, l'intérêt de mettre cette hypothèse en exergue, comme conduisent à le faire les résultats de cette étude, est de prendre en défaut l'idée trop simpliste que les OEA étant le reflet de phénomènes actifs cochléaires, et ces phénomènes étant hautement sélectifs en fréquence, toutes les propriétés des OEA manifestent cette sélectivité. Notamment, le fait d'utiliser un stimulus lui-même sélectif en fréquence (pour détecter les SFE) ne suffit pas à garantir l'indépendance des SFE vis-à-vis de l'état global de la cochlée. Ce résultat rejoint celui publié récemment par Guinan [9] qui démontre que les SFE sont sensibles à des actions du système efférent médian même si ces actions sont supposées n'agir que sur d'autres fréquences. Une conséquence indirecte des résultats du présent travail est l'impossibilité d'utiliser les otoémissions provoquées (en tout cas les paramètres étudiés ici) pour le diagnostic précoce des lésions dues aux sons forts, puisque les modifications des OEA sont le reflet de lésions basales déjà étendues.

\section{Bibliographie}

[1] Gold T. (1988) Historical background to the proposal, 40 years ago, of an active model for cochlear frequency analysis. In: J.P.Wilson, D.T.Kemp (Eds), Cochlear mechanisms, Structures, Functions and Models, Plenum Press, pp.299-305.

[2] Avan P., Bonfils P., Loth D., Narcy P. and Trotoux J. (1991) Quantitative assessment of human cochlear function by evoked otoacoustic emissions. Hear. Res., 52, 99-112.

[3] Bonfils P., Dumont A., Marie P., François M. and Narcy P. (1990) Evoked otoacoustic emissions in newborn hearing screening. Laryngoscope, 100, 186-189.

[4] Kemp D.T., Ryan S. and Bray P. (1990) A guide to the effective use of otoacoustic emissions. Ear Hear.11, 93-105.

[5] Sutton G.J. and Wilson J.P. (1983) Modelling cochlear echoes: the influence of irregularities in frequency mapping on summed cochlear activity. In: E.de Boer, M.A.Viergever (Eds), Mechanisms of Hearing, Delft Univ.Press, Delft, The Netherlands, pp.83-90.

[6] Zwicker E. (1989) Otoacoustic emissions and cochlear travelling waves. In: J.P.Wilson, D.T.Kemp (Eds), Cochlear mechanisms, structures, function and models, Plenum Press, pp.359-366.

[7] Strube H.W. (1989) Evoked otoacoustic emissions as cochlear Bragg reflections. Hear. Res.38, 3546.

[8] Inselberg A. (1978) Cochlear dynamics: the evolution of a mathematical model. SIAM review, 20, 301-351.

[9] Guinan J.J.Jr. (1990) Changes in stimulus frequency otoacoustic emissions produced by two-tone suppression and efferent stimulation in cats. In: P.Dallos, C.D.Geisler, J.W.Matthews, C.R.Steele(Eds), Mechanics and biophysics of hearing, Springer Verlag, pp.170-177.

(Travail partiellement financé par le Ministère de l'Environnement, SRETIE, Contrat $n^{\circ} 89090$ ) 